\title{
INTRODUCTION TO COCHAINS OF DIFFERENTIAL OPERATORS
}

\author{
F. J. BLOORE and G. ROBERTS \\ Department of Mathematical Sciences, Liverpool University, Liverpool L69 3BX, England \\ E-mail: f.j.bloore@liverpool.ac.uk
}

To Professor W. M. Tulczyjew on the occasion of his 70th birthday

I first heard a lecture by Włodek Tulczyjew at Aix en Provence in 1979, and I remember it. I had been struggling for some time to grasp the basics of spectral sequences in order to understand the topological approach to the statistics of identical particles. Here spoke a physicist who was completely on top of the technology - using it to place the Euler-Lagrange operator in an exact sequence, to provide a test of whether or not a given set of differential equations arose from a Lagrangian. And he even said that this was not his main research interest, but something he did in odd moments! So I was impressed. Many happy returns.

Fred Bloore

1. Introduction. The modern treatment of mechanics, initiated and developed by Tulczyjew and others, uses the differential geometry associated with the tangent and cotangent bundles of the configuration space $Q$ of the mechanical system. Its tools are the vector fields and the differential forms dual to them together with their exterior derivatives.

This theory has a rich structure and enjoys great success in describing mechanical systems. So it is not surprising that less attention has been paid to the fact that, since vector fields may be viewed as first order linear differential operators on the set $\mathcal{F}=C^{\infty}(Q, \mathbf{C})$ of smooth functions on $Q$, there may be some reward for studying the linear differential operators of higher order by means of their cochains, in the same spirit that we study the vector fields through their differential forms. (We choose $\mathbf{C}$ rather than $\mathbf{R}$ because one application (section 6.1) requires quantum mechanical wave functions to lie in $\mathcal{F}$.)

In this contribution we offer a start on this study. (It's not our first paper on the stuff but we hope it is a gateway into the field.) There is also a well developed jet bundle theory

2000 Mathematics Subject Classification: 16E40, 58A99.

The paper is in final form and no version of it will be published elsewhere. 
of systems of partial differential equations begun by Cartan, Kaehler, Janet, Riquier, Spencer with which the present material is not yet linked.

We shall study the associative algebra $\mathcal{D}$ of linear differential operators on $\mathcal{F}$ by exploring the properties of the Hochschild complex of $\mathcal{D}$-valued cochains on $\mathcal{D}$. This complex has two interesting subcomplexes, the complex of $\mathcal{F}$-tensorial cochains and its normalised subcomplex, the $\mathcal{F}$-relative cochains. These latter are our principal object of concern. When restricted to act on vector fields they reduce to covariant tensor fields. They may be regarded as the natural extension of covariant tensor fields (and of differential forms in particular) to act on differential operators of order higher than one.

Section 2 contains the definitions and basic properties. The following section gives a local characterisation of $\mathcal{F}$-tensorial cochains in terms of their "structure functions". In section 4 we sketch out a useful isomorphism of differential graded algebras which relates an $\mathcal{F}$-tensorial $p$-cochain on $\mathcal{D}$ to the jet of a smooth function on $Q^{p+1}$ taken on the diagonal. We take the small liberty of calling the complex of these jets the AlexanderSpanier complex - the two notions are so similar. In section 5 we show a rather natural way to enlist the aid of a connection on $T Q$ to extend the domain of a differential form to include all $\mathcal{D}$. Some possible uses of these cochains are suggested in Section 6.

2. Cochains on $\mathcal{D}$. We first define a general $\mathcal{D}$-valued Hochschild cochain, then specialise to an $\mathcal{F}$-tensorial cochain. Our main objects of study, the $\mathcal{F}$-relative cochains, are simply the normalised $\mathcal{F}$-tensorial ones. Each type has a differential algebra using the Hochschild differential $\delta$ and the cup product $\cup$. We define also the composition $\circ$ of a 1-cochain with a $p$-cochain.

When an $\mathcal{F}$-relative $p$-cochain is restricted to act only on vector fields it reduces to a covariant tensor field whose anti-symmetric part is a de Rham differential $p$-form. The consequent map a from $\mathcal{F}$-relative cochains on $\mathcal{D}$ to de Rham differential forms respects the differential structure. This motivates the investigation of how much of the existing structure $[\mathrm{CP}]$ of differential forms and vector fields can be carried back to Hochschild cochains and linear differential operators.

We define, for any linear differential operator $K$, the interior product $\iota_{K} A$ and the Lie derivative $L_{K} A$ of a general Hochschild cochain $A$ on $\mathcal{D}$. We then show, mainly by direct calculation, that almost all the classical properties of de Rham forms in regard to $\iota_{X}$ and $L_{X}, X \in \mathcal{X}(Q)$, are mirrored by properties of cochains in regard to $\iota_{K}$ and $L_{K}$. These include the Cartan homotopy relation

$$
d \iota_{X} \omega+\iota_{X} d \omega=L_{X} \omega .
$$

2.1. Hochschild p-cochains on $\mathcal{D}$

Definition. A Hochschild p-cochain on $\mathcal{D}$ is a $\mathbf{C}$-linear map

$$
A: \mathcal{D} \otimes \mathcal{D} \otimes \ldots \otimes \mathcal{D} \rightarrow \mathcal{D}
$$

with $p$ factors in the tensor product. Here (and throughout) $\otimes$ will denote $\otimes_{\mathbf{C}}$. A 0 -cochain is an element of $\mathcal{D}$.

The set of Hochschild $p$-cochains on $\mathcal{D}$ will be denoted by $C^{p}(\mathcal{D}, \mathcal{D})$. 
Definition [Ho1]. For $A \in C^{p}(\mathcal{D}, \mathcal{D})$ the Hochschild differential $\delta A$ is the $(p+1)$ cochain defined, for $H_{i} \in \mathcal{D}$, by

$$
\begin{aligned}
& (\delta A)\left(H_{1}, \ldots, H_{p+1}\right)=H_{1} A\left(H_{2}, \ldots, H_{p+1}\right)-A\left(H_{1} H_{2}, \ldots, H_{p+1}\right) \\
& +\ldots+(-1)^{p} A\left(H_{1}, \ldots, H_{p} H_{p+1}\right)+(-1)^{p+1} A\left(H_{1}, \ldots, H_{p}\right) H_{p+1} .
\end{aligned}
$$

So in particular, for $H \in C^{0}(\mathcal{D}, \mathcal{D}), K \in \mathcal{D}\left(=C^{0}(\mathcal{D}, \mathcal{D})\right)$,

$$
\delta H(K)=[K, H]=K H-H K .
$$

Definition. For $A \in C^{p}(\mathcal{D}, \mathcal{D}), B \in C^{q}(\mathcal{D}, \mathcal{D})$, the cup product $A \cup B \in C^{p+q}(\mathcal{D}, \mathcal{D})$ is defined by

$$
(A \cup B)\left(H_{1}, \ldots, H_{p+q}\right)=A\left(H_{1}, \ldots, H_{p}\right) \circ B\left(H_{p+1}, \ldots, H_{p+q}\right)
$$

where $\circ$ is the composition of differential operators.

It follows from the definitions that

$$
\delta(A \cup B)=\delta A \cup B+(-1)^{p} A \cup \delta B .
$$

Note that for $H_{1}, H_{2} \in C^{0}(\mathcal{D}, \mathcal{D})=\mathcal{D}$,

$$
H_{1} \cup H_{2}=H_{1} H_{2} \text {. }
$$

For the particular case of $A \in C^{1}(\mathcal{D}, \mathcal{D})$ and $B \in C^{p}(\mathcal{D}, \mathcal{D})$ we also have the composition $A \circ B \in C^{p}(\mathcal{D}, \mathcal{D})$ :

$$
(A \circ B)\left(H_{1}, \ldots, H_{p}\right)=A\left(B\left(H_{1}, \ldots, H_{p}\right)\right) .
$$

Definition. For $p \geq 1, A \in C^{p}(\mathcal{D}, \mathcal{D})$ is called normalised if $A\left(H_{1}, \ldots, H_{p}\right)=0$ whenever one or more $H_{j}$ is the identity operator in $\mathcal{D}$.

If $A$ and $B$ are normalised so are $\delta A, A \cup B$ and, for $A \in C^{1}(\mathcal{D}, \mathcal{D}), A \circ B$ is normalised whenever $B$ is normalised.

From now on we shall simply write the word cochains for Hochschild cochains on $\mathcal{D}$ and assume that all differential operators mentioned are linear.

2.2. $\mathcal{F}$-tensorial p-cochains

Definition. A cochain $A \in C^{p}(\mathcal{D}, \mathcal{D})$ is $\mathcal{F}$-tensorial if for $H_{i} \in \mathcal{D}, f_{j} \in \mathcal{F}$,

$$
A\left(f_{1} H_{1}, f_{2} H_{2}, f_{3} H_{3}, \ldots, f_{p} H_{p} f_{p+1}\right)=f_{1} \circ A\left(H_{1} \circ f_{2}, H_{2} \circ f_{3}, \ldots, H_{p}\right) \circ f_{p+1}
$$

i.e. the $f_{j}$ can jump between the $H_{j}$ but not through them (except when $H_{j} \in \mathcal{F}$ ). The set of $\mathcal{F}$-tensorial $p$-cochains is denoted by $C_{\mathcal{F}}^{p}(\mathcal{D}, \mathcal{D})$.

The algebra $\mathcal{D}$ is filtered by order; we shall denote by $\mathcal{D}_{p}$ the subset of operators with order $p$ or less.

An $\mathcal{F}$-tensorial 0-cochain is taken to be an element of $\mathcal{F}=\mathcal{D}_{0} \subset \mathcal{D}$, the commutative subalgebra of differential operators of zero order. This is to ensure that $\delta$ preserves the $\mathcal{F}$-tensorial property: For $H \in C^{0}(\mathcal{D}, \mathcal{D})=\mathcal{D}$, we note that $\delta H \in C_{\mathcal{F}}^{1}(\mathcal{D}, \mathcal{D})$ only if $\delta H(f K)=f \delta H(K)$ for all $f \in \mathcal{F}, K \in \mathcal{D}$. But this implies $[H, f]=0$, so we need $H \in \mathcal{F}$.

Definition. A $p$-cochain is said to be $\mathcal{F}$-relative [Ho2] if it is both $\mathcal{F}$-tensorial and normalised. 
It follows that if $A$ is $\mathcal{F}$-relative then

$$
A\left(H_{1}, \ldots, H_{p}\right)=0
$$

whenever one or more $H_{j} \in \mathcal{F}$. The set of $\mathcal{F}$-relative cochains is denoted by $C^{p}(\mathcal{D}, \mathcal{F} ; \mathcal{D})$. And $C^{0}(\mathcal{D}, \mathcal{F} ; \mathcal{D})=C_{\mathcal{F}}^{0}(\mathcal{D}, \mathcal{D})=\mathcal{F}$.

If $A$ and $B$ are $\mathcal{F}$-tensorial $(\mathcal{F}$-relative) then so are $\delta A, A \cup B$ and, when defined, $A \circ B$.

We shall see in section 3 that the composition product of $\mathcal{F}$-tensorial 1 -cochains is commutative.

2.3. The restriction of $\mathcal{F}$-relative cochains to vector fields. Consider an $\mathcal{F}$-relative 2-cochain $A$ acting on vector fields $X_{1}, X_{2}$ :

$$
A\left(X_{1}, X_{2}\right) \in \mathcal{D} \text {. }
$$

For $f \in \mathcal{D}_{0}=\mathcal{F}$,

$$
\begin{aligned}
A\left(X_{1}, X_{2}\right) \circ f & =A\left(X_{1}, X_{2} \circ f\right) & & \text { by } \mathcal{F} \text {-tensoriality } \\
& =A\left(X_{1},\left[X_{2}, f\right]+f X_{2}\right) & & \\
& =A\left(X_{1}, f X_{2}\right) & & \text { by normalisation and linearity } \\
& =A\left(X_{1} \circ f, X_{2}\right) & & \text { by } \mathcal{F} \text {-tensoriality } \\
& =A\left(\left[X_{1}, f\right]+f X_{1}, X_{2}\right) & & \\
& =A\left(f X_{1}, X_{2}\right) & & \text { by normalisation } \\
& =f \circ A\left(X_{1}, X_{2}\right) & & \text { by } \mathcal{F} \text {-tensoriality. }
\end{aligned}
$$

So

$$
A\left(X_{1}, X_{2}\right) \circ f-f \circ A\left(X_{1}, X_{2}\right)=\left[A\left(X_{1}, X_{2}\right), f\right]=0,
$$

and therefore

$$
A\left(X_{1}, X_{2}\right) \in \mathcal{F}
$$

A similar argument yields

$$
A\left(f_{1} X_{1}, f_{2} X_{2}\right)=f_{1} f_{2} A\left(X_{1}, X_{2}\right),
$$

telling us that $\left.A\right|_{\mathcal{X}}$ is a $(0,2)$ tensor field, and indeed for $A \in C^{p}(\mathcal{D}, \mathcal{F} ; \mathcal{D})$, we have that $\left.A\right|_{\mathcal{X}}$ is a $(0, p)$ tensor field.

Let ord $H$ denote the order of the differential operator $H$. One can show $[\mathrm{HB}]$ that for $A \in C^{p}(\mathcal{D}, \mathcal{F} ; \mathcal{D})$ and $H_{j} \in \mathcal{D}$ with ord $H_{j}=k_{j}$ then

$$
\operatorname{ord}\left(A\left(H_{1}, \ldots, H_{p}\right)\right)= \begin{cases}\sum_{j=1}^{p}\left(k_{j}-1\right) & \text { if all } k_{j} \neq 0 \\ 0 & \text { if any } k_{j}=0\end{cases}
$$

Let us define the map $a$ from $\mathcal{F}$-relative cochains to differential forms by

$$
\begin{gathered}
a: C^{p}(\mathcal{D}, \mathcal{F} ; \mathcal{D}) \rightarrow \Omega^{p}(Q, \mathbf{C}), \\
(a A)\left(X_{1}, \ldots, X_{p}\right)=\sum_{\sigma \in \mathcal{S}_{p}}(\operatorname{sgn} \sigma) A\left(X_{\sigma_{1}}, \ldots, X_{\sigma_{p}}\right),
\end{gathered}
$$

where $X_{j} \in \mathcal{X}$ and where $\mathcal{S}_{p}$ is the symmetric group. That is to say, $a$ is the restriction of $C^{p}(\mathcal{D}, \mathcal{F} ; \mathcal{D})$ to vector fields, followed by anti-symmetrisation. Direct calculation reveals that

$$
a \circ \delta=d \circ a \quad \text { and } \quad a(A \cup B)=(a A) \wedge(a B)
$$


Thus $a$ is in fact a morphism of the differential graded algebras:

$$
a:\left(C^{*}(\mathcal{D}, \mathcal{F} ; \mathcal{D}), \delta, \cup\right) \rightarrow\left(\Omega^{*}(Q, \mathbf{C}), d, \wedge\right) .
$$

2.4. Interior product and Lie derivative. Vector fields $X$ and differential forms $\omega$ possess a rich algebraic structure based on the interior product $\iota_{V} \omega$, the Lie derivative $L_{V} \omega$, the coboundary operator $d$ and the wedge product $\wedge[\mathrm{CP}]$. Almost all this structure is inherited from corresponding structure on differential operators and cochains, (general cochains, not just $\mathcal{F}$-tensorial ones). The definitions and calculations for the general cochains make no use of the properties of the linear differential operators. The proofs of equations (5), (6) and of properties [1], [2], [3], [5], [6], [8] are combinatoric and could be applied equally to any cochains on any associative algebra with unit. Only the statements regarding $\mathcal{F}$-tensorial or $\mathcal{F}$-relative cochains are specific to $\mathcal{D}$.

\subsubsection{Interior product}

Definition. For $H_{i}, K \in \mathcal{D}$, the interior product

$$
\iota_{K}: C^{p+1}(\mathcal{D}, \mathcal{D}) \rightarrow C^{p}(\mathcal{D}, \mathcal{D})
$$

is given by

$$
\begin{aligned}
\left(\iota_{K} A\right)\left(H_{1}, \ldots, H_{p}\right)= & A\left(K, H_{1}, \ldots, H_{p}\right)+\sum_{k=1}^{p-1}(-1)^{k} A\left(H_{1}, \ldots, H_{k}, K, H_{k+1}, \ldots, H_{p}\right) \\
& +(-1)^{p} A\left(H_{1}, \ldots, H_{p}, K\right) .
\end{aligned}
$$

Then by straightforward calculation

$$
\iota_{K}(A \cup B)=\left(\iota_{K} A\right) \cup B+(-1)^{\operatorname{deg} A} A \cup\left(\iota_{K} B\right)
$$

and

$$
\iota_{K}^{2}=0 .
$$

If $A \in C^{p}(\mathcal{D}, \mathcal{D})$ is normalised then so also is $\iota_{K} A$.

If $A$ is $\mathcal{F}$-tensorial then $\iota_{K} A$ is $\mathcal{F}$-tensorial if and only if $K \in \mathcal{F}$.

If $A$ is $\mathcal{F}$-relative then $\iota_{K} A$ is $\mathcal{F}$-relative if and only if ord $K \leq 1$.

\subsubsection{Lie derivative}

Definition. For $K, H \in \mathcal{D}$ we define the Lie derivative of $H$ by $K$ to be

$$
L_{K} H=[K, H] \text {. }
$$

Definition. For $A \in C^{p}(\mathcal{D}, \mathcal{D}), L_{K} A \in C^{p}(\mathcal{D}, \mathcal{D})$ is given by

$$
\left(L_{K} A\right)\left(H_{1}, \ldots, H_{p}\right)=\left[K, A\left(H_{1}, \ldots, H_{p}\right)\right]-\sum_{k=1}^{p} A\left(H_{1}, \ldots,\left[K, H_{k}\right], \ldots, H_{p}\right) .
$$

Note that for $H \in C^{0}(\mathcal{D}, \mathcal{D})=\mathcal{D}$ we have $L_{K} H=[K, H]$. The action of $L_{K}$ is the same whether you regard $H$ as a cochain or a linear differential operator.

If $A \in C^{p}(\mathcal{D}, \mathcal{D})$ is normalised then so is $L_{K} A$.

If $A$ is $\mathcal{F}$-tensorial $\left(\mathcal{F}\right.$-relative) then $L_{K}$ is $\mathcal{F}$-tensorial $(\mathcal{F}$-relative) if and only if ord $K \leq 1$. 
2.4.3. Properties of Lie derivative and interior product. Let $K_{i}, H_{j} \in \mathcal{D}$, let $f \in \mathcal{F}$, and let $A \in C^{p}(\mathcal{D}, \mathcal{D}), B \in C^{q}(\mathcal{D}, \mathcal{D})$. The following properties [1]-[4] are trivial.

$$
\begin{aligned}
L_{K}\left(H_{1} H_{2}\right) & =\left(L_{K} H_{1}\right) H_{2}+H_{1}\left(L_{K} H_{2}\right), \\
L_{K_{1} K_{2}} H & =\left(L_{K_{1}} H\right) K_{2}+K_{1}\left(L_{K_{2}} H\right), \\
L_{K}(A \cup B) & =\left(L_{K} A\right) \cup B+A \cup\left(L_{K} B\right), \\
L_{K}(f A) & =\left(L_{K} f\right) A+f L_{K} A .
\end{aligned}
$$

Property [4] is a special case of [3], since $f \in C^{0}(\mathcal{D}, \mathcal{D})$ and $f A=f \cup A$.

A bit less trivial is

$$
L_{\left[K_{1}, K_{2}\right]} A=L_{K_{1}} L_{K_{2}} A-L_{K_{2}} L_{K_{1}} A \equiv\left[L_{K_{1}}, L_{K_{2}}\right] A .
$$

Proof of [5]: We adopt the notation

$$
\underline{H}=H_{1} \otimes \ldots \otimes H_{p}
$$

and call it a $p$-chain. Denote the space of $p$-chains as $C_{p}(\mathcal{D})$. Write also

$$
[K, \underline{H}]=-[\underline{H}, K]=\sum_{k=1}^{p}\left(H_{1} \otimes \ldots \otimes\left[K, H_{k}\right] \otimes \ldots \otimes H_{p}\right) .
$$

Then

$$
\left(L_{K} A\right)(\underline{H})=[K, A(\underline{H})]-A([K, \underline{H}])
$$

and

$$
\left[K_{1},\left[K_{2}, \underline{H}\right]\right]+\left[K_{2},\left[\underline{H}, K_{1}\right]\right]+\left[\underline{H},\left[K_{1}, K_{2}\right]\right]=0 \text {. }
$$

So

$$
\begin{aligned}
L_{K_{1}} L_{K_{2}} A(\underline{H}) & =\left[K_{1},\left(L_{K_{2}} A\right)(\underline{H})\right]-\left(L_{K_{2}} A\right)\left(\left[K_{1}, \underline{H}\right]\right) \\
= & {\left[K_{1},\left[K_{2}, A(\underline{H})\right]\right]-\left[K_{1}, A\left(\left[K_{2}, \underline{H}\right]\right)\right]-\left[K_{2}, A\left(\left[K_{1}, \underline{H}\right]\right)\right]+A\left(\left[K_{2},\left[K_{1}, \underline{H}\right]\right]\right) }
\end{aligned}
$$

whence the result.

For a $p$-form $\alpha$ and vector fields $V, W$ we have

$$
\begin{aligned}
\iota_{[V, W]} \alpha & =L_{V} \iota_{W} \alpha-\iota_{W} L_{V} \alpha, \\
L_{f V} \alpha & =f L_{V} \alpha+d f \wedge\left(\iota_{V} \alpha\right) .
\end{aligned}
$$

Direct calculation yields the corresponding cochain version of $\left[6^{\prime}\right]$,

[6]

$$
\iota_{\left[K_{1}, K_{2}\right]} A=L_{K_{1}} \iota_{K_{2}} A-\iota_{K_{2}} L_{K_{1}} A .
$$

Identity $\left[7^{\prime}\right]$ does not have a simple generalisation to cochains.

2.4.4. The Cartan identity. The Cartan relation in differential geometry, $\left[8^{\prime}\right]$

$$
L_{X}=d \iota_{X}+\iota_{X} d
$$

extends to cochains in the form

$$
L_{K}=\delta \iota_{K}+\iota_{K} \delta
$$

Proof. It is helpful first to install some more notation. We have from equation (9)

$$
L_{K}(A(\underline{H}))=\left(L_{K} A\right)(\underline{H})+A\left(L_{K} \underline{H}\right) .
$$


The operators $\iota_{K}$ and $\delta$ have so far been defined only on cochains. Let us define them on chains also, $\iota_{K}: C_{p} \rightarrow C_{p+1}, \quad \partial: C_{p} \rightarrow C_{p-1}$, by

$$
\begin{aligned}
\iota_{K} \underline{H}= & K \otimes \underline{H}+\sum_{j=1}^{p}(-1)^{j} H_{1} \otimes \ldots \otimes H_{j} \otimes K \otimes H_{j+1} \otimes \ldots \otimes H_{p}, \\
\partial \underline{H}= & H_{1} H_{2} \otimes H_{3} \otimes \ldots \otimes H_{p}-H_{1} \otimes H_{2} H_{3} \otimes H_{4} \otimes \ldots \otimes H_{p}+\ldots \\
& +(-1)^{p} H_{1} \otimes \ldots \otimes H_{p-1} H_{p} .
\end{aligned}
$$

Then

$$
\iota_{K} A=A \circ \iota_{K} \text { and } \delta A+A \circ \partial=1 \cup A+(-1)^{p+1} A \cup 1
$$

where $A \in C^{p}(\mathcal{D}, \mathcal{D})$ and $1 \in C^{1}(\mathcal{D}, \mathcal{D})$ denotes the identity 1-cochain whose value on $H$ is $H$. On products we have

$$
\begin{gathered}
\iota_{K}\left(\underline{H} \otimes \underline{H}^{\prime}\right)=\left(\iota_{K} \underline{H}\right) \otimes \underline{H}^{\prime}+(-1)^{\operatorname{deg} \underline{H}} \underline{H} \otimes \iota_{K} \underline{H}^{\prime}-(-1)^{\operatorname{deg} \underline{H}} \underline{H} \otimes K \otimes \underline{H}^{\prime}, \\
\partial\left(\underline{H} \otimes \underline{H}^{\prime}\right)=(\partial \underline{H}) \otimes \underline{H}^{\prime}+(-1)^{\operatorname{deg}} \underline{H} \underline{H} \otimes \partial \underline{H}^{\prime}-(-1)^{\operatorname{deg} \underline{H}} \underline{H} \cdot \underline{H}^{\prime}
\end{gathered}
$$

where the concatenation product [Coq] of chains is

$$
\underline{H} \cdot \underline{H}^{\prime}=H_{1} \otimes \ldots \otimes H_{p} H_{1}^{\prime} \otimes H_{2}^{\prime} \otimes \ldots \otimes H_{q}^{\prime} .
$$

LEMMA. $\left(\partial \iota_{K}+\iota_{K} \partial\right) \underline{H}=L_{K}(\underline{H})$.

Proof. On the right, the map $\underline{H} \mapsto L_{K}(\underline{H})=[K, \underline{H}]$ is a derivation over $\otimes$. It is easy to check that the lemma holds on 1-chains, and also that $\partial \iota_{K}+\iota_{K} \partial$ is a derivation over $\otimes$.

Returning to [8], the terms on the right side may be written

$$
\begin{gathered}
\left(\delta\left(\iota_{K} A\right)\right)(\underline{H})=\left(1 \cup\left(\iota_{K} A\right)-\left(\iota_{K} A\right) \circ \partial+(-1)^{p}\left(\iota_{K} A\right) \cup 1\right)(\underline{H}), \\
\left(\iota_{K}(\delta A)\right)(\underline{H})=(\delta A)\left(\iota_{K}(\underline{H})\right)=\left(1 \cup A-A \circ \partial+(-1)^{p+1} A \cup 1\right)\left(\iota_{K}(\underline{H})\right) .
\end{gathered}
$$

The first terms of the right sides of these two equations add to give $K A(\underline{H})$. The last terms add to give $-A(\underline{H}) K$. The middle terms add, by the lemma, to give $-A([K, \underline{H}])$. Hence [8].

\section{The structure of $\mathcal{F}$-tensorial cochains}

\subsection{Introduction}

Notation. For local coordinates $x^{i}$ on $Q$ let $I=\left\{i_{1}, \ldots, i_{|I|}\right\}$ be an unordered set of indices, and write

$$
\partial_{i}=\frac{\partial}{\partial x^{i}}, \quad \partial_{I}=\partial_{i_{1}} \partial_{i_{2}} \ldots \partial_{i_{|I|}}=\prod_{i \in I} \partial_{i}, \quad f_{, I}=\partial_{I} f
$$

In the case $I=\emptyset$, the empty set, we shall mean by $\partial_{\emptyset}$ the unit operator. It is a differential operator of zero order, and may be identified with the unit function 1 on $Q$.

With this notation the Leibniz rule for derivatives of products of functions may be written

$$
(f g)_{, I}=\sum_{I_{1} \cup I_{2}=I} f_{, I_{1}} g_{, I_{2}}
$$


with the sum taken over all partitions of $I$ including $(\emptyset, I)$ and $(I, \emptyset)$. It is understood that $I_{1} \cap I_{2}=\emptyset$.

In section 3.2 it is shown that any $\mathcal{F}$-tensorial $p$-cochain $A$ is uniquely characterised, in local coordinates, by its "structure functions" $A_{\left(I_{1}, \ldots, I_{p}\right)}$ where $A_{\left(I_{1}, \ldots, I_{p}\right)}(x)=$ $\left.A\left(\partial_{I_{1}}, \ldots, \partial_{I_{p}}\right) 1\right|_{x}$ is the zero order term of the differential operator $A\left(\partial_{I_{1}}, \ldots, \partial_{I_{p}}\right)$ evaluated at the point $x \in Q$.

In section 3.3 are computed the structure functions of $\delta A, A \cup B$, the composition product $\theta \circ \theta^{\prime}$ of two 1 -cochains and the structure functions of normalised $\mathcal{F}$-tensorial cochains. Those of $\theta \circ \theta^{\prime}$ are used to prove that composition of such 1-cochains is commutative.

In section 3.4 the structure functions are used to express $A$ in terms of a Taylor-like series of "basis" cochains $\delta x^{I_{1}} \cup \ldots \cup \delta x^{I_{p}}$. This is the cochain version of the expression of a $p$-form in terms of wedge products of coordinate differentials, $d x^{i_{1}} \wedge \ldots \wedge d x^{i_{p}}$.

3.2. The structure theorem for $\mathcal{F}$-tensorial cochains

(i) Consider the example of $A \in C_{\mathcal{F}}^{2}(\mathcal{D}, \mathcal{D})$. Let $H, K \in \mathcal{D}$,

$$
H=h^{i_{1} \ldots i_{|I|}} \partial_{i_{1}} \ldots \partial_{i_{|I|}} \equiv h^{I} \partial_{I}, \quad K=k^{J} \partial_{J}
$$

where any repeated index $i_{l}$ is summed. Then

$$
\begin{array}{rlrl}
A\left(h^{I} \partial_{I}, k^{J} \partial_{J}\right) & =h^{I} A\left(\partial_{I} \circ k^{J}, \partial_{J}\right) & & \text { using } \mathcal{F} \text {-tensoriality, } \\
& =h^{I} A\left(\sum_{I_{1} \cup I_{2}=I} k_{, I_{1}}^{J} \partial_{I_{2}}, \partial_{J}\right) & & \text { using Leibniz' rule, } \\
& =\sum_{I_{1} \cup I_{2}=I} h^{I} k_{, I_{1}}^{J} A\left(\partial_{I_{2}}, \partial_{J}\right) & \text { using } \mathcal{F} \text {-tensoriality. }
\end{array}
$$

For any $A \in C_{\mathcal{F}}^{p}(\mathcal{D}, \mathcal{D})$ we can cascade the functions to the front as in the above example. Thus $A$ is completely determined by its action on the $\partial_{I}$, the basis elements of $\mathcal{D}$ in the coordinate neighbourhood.

(ii) Let $\psi \in \mathcal{F}$ be the target function for the differential operator $A\left(\partial_{I}, \partial_{J}\right)$. Then, with 1 denoting the unit function,

$$
\begin{aligned}
A\left(\partial_{I}, \partial_{J}\right) \psi & =A\left(\partial_{I}, \partial_{J} \circ \psi\right) 1 \\
& =\sum_{J_{1} \cup J_{2}=J} A\left(\partial_{I} \circ \psi_{, J_{1}}, \partial_{J_{2}}\right) 1 \\
& =\sum_{I_{1} \cup I_{2}=I} \sum_{J_{1} \cup J_{2}=J} \psi_{, I_{1} \cup J_{1}} A\left(\partial_{I_{2}}, \partial_{J_{2}}\right) 1 \\
& =\sum_{I_{1} \cup I_{2}=I} \sum_{J_{1} \cup J_{2}=J}\left(A\left(\partial_{I_{2}}, \partial_{J_{2}}\right) 1\right) \psi_{, I_{1} \cup J_{1}}
\end{aligned}
$$

The same argument for $p$-cochains gives $[\mathrm{BR}]$

$$
A\left(\partial_{I_{1}}, \ldots, \partial_{I_{p}}\right)=\sum_{I_{11} \cup I_{12}=I_{1}} \ldots \sum_{I_{p 1} \cup I_{p 2}=I_{p}} A_{\left(I_{12}, \ldots, I_{p 2}\right)} \partial_{I_{11} \cup \ldots \cup I_{p 1}}
$$

where we have written

$$
A\left(\partial_{I_{12}}, \ldots, \partial_{I_{p 2}}\right) 1=A_{\left(I_{12}, \ldots, I_{p 2}\right)} .
$$


The functions $A_{\left(J_{1}, \ldots, J_{p}\right)}$ are called the structure functions of the cochain $A$. They evidently characterise $A$ on the coordinate patch.

Two low order examples are:

If $A \in C_{\mathcal{F}}^{1}(\mathcal{D}, \mathcal{D})$ then

$$
\begin{gathered}
A\left(\partial_{i}\right)=A_{(i)}+A_{(\emptyset)} \partial_{i} \\
A\left(\partial_{i} \partial_{j}\right)=A_{(i j)}+A_{(i)} \partial_{j}+A_{(j)} \partial_{i}+A_{(\emptyset)} \partial_{i} \partial_{j} \\
A\left(\partial_{i} \partial_{j} \partial_{k}\right)=A_{(i j k)}+A_{(i j)} \partial_{k}+A_{(j k)} \partial_{i}+A_{(k i)} \partial_{j}+A_{(i)} \partial_{j} \partial_{k} \\
+A_{(j)} \partial_{k} \partial_{i}+A_{(k)} \partial_{i} \partial_{j}+A_{(\emptyset)} \partial_{i} \partial_{j} \partial_{k} .
\end{gathered}
$$

If $A \in C_{\mathcal{F}}^{2}(\mathcal{D}, \mathcal{D})$, then

$$
\begin{aligned}
A\left(\partial_{i}, \partial_{j} \partial_{k}\right)= & A_{(i, j k)}+A_{(\emptyset, j k)} \partial_{i}+A_{(i, k)} \partial_{j}+A_{(i, j)} \partial_{k} \\
& +A_{(\emptyset, k)} \partial_{i} \partial_{j}+A_{(\emptyset, j)} \partial_{i} \partial_{k}+A_{(i, \emptyset)} \partial_{j} \partial_{k}+A_{(\emptyset, \emptyset)} \partial_{i} \partial_{j} \partial_{k} .
\end{aligned}
$$

3.3. The structure functions for $\delta A, A \cup B, \theta \circ \theta^{\prime}$ and for normalised $\mathcal{F}$-tensorial cochains. Let $A \in C_{\mathcal{F}}^{p}(\mathcal{D}, \mathcal{D}), \quad B \in C_{\mathcal{F}}^{q}(\mathcal{D}, \mathcal{D})$. The following results are easy.

(i) $(\delta A)_{\left(I_{1}, \ldots, I_{p+1}\right)}=A_{\left(I_{2}, \ldots, I_{p+1}\right), I_{1}}-A_{\partial\left(I_{1}, \ldots, I_{p+1}\right)}+(-1)^{p+1} A_{\left(I_{1}, \ldots, I_{p}\right)} \delta_{I_{p+1}, \emptyset}$ where

$$
\begin{aligned}
\partial\left(I_{1}, \ldots, I_{p+1}\right) \equiv & \left(I_{1} \cup I_{2}, \ldots, I_{p+1}\right)-\left(I_{1}, I_{2} \cup I_{3}, \ldots, I_{p+1}\right) \\
& +\ldots+(-1)^{p+1}\left(I_{1}, \ldots, I_{p} \cup I_{p+1}\right),
\end{aligned}
$$

(a formal sum of $p$-tuples of sets of indices),

$$
\delta_{I_{p+1}, \emptyset}= \begin{cases}0 & \text { if } I_{p+1} \neq \emptyset \\ 1 & \text { if } I_{p+1}=\emptyset\end{cases}
$$

and $A_{\left(I_{1}, \ldots, I_{p}\right)+\left(J_{1}, \ldots, J_{p}\right)}=A_{\left(I_{1}, \ldots, I_{p}\right)}+A_{\left(J_{1}, \ldots, J_{p}\right)}$.

(ii) $(A \cup B)_{\left(I_{1}, \ldots, I_{p+q}\right)}=\sum_{I_{11} \cup I_{12}=I_{1}} \ldots \sum_{I_{p 1} \cup I_{p 2}=I_{p}} A_{\left(I_{11}, \ldots, I_{p 1}\right)} B_{\left(I_{p+1}, \ldots, I_{p+q}\right), I_{12} \cup \ldots \cup I_{p 2}}$.

(iii) For $\theta, \theta^{\prime} \in C_{\mathcal{F}}^{1}(\mathcal{D}, \mathcal{D})$ we have

$$
\left(\theta \circ \theta^{\prime}\right)_{(I)}=\sum_{I_{1} \cup I_{2}=I} \theta_{\left(I_{2}\right)}^{\prime} \theta_{\left(I_{1}\right)} .
$$

This is the same as $\left(\theta^{\prime} \circ \theta\right)_{(I)}$. Hence $\theta^{\prime} \circ \theta=\theta \circ \theta^{\prime}$ and the composition of $\mathcal{F}$-tensorial 1-cochains is commutative.

(iv) An $\mathcal{F}$-tensorial $p$-cochain $A$ is normalised, i.e. $\mathcal{F}$-relative if and only if $A_{\left(I_{1}, \ldots, I_{p}\right)}=$ 0 whenever at least one of the $I_{k}=\emptyset$.

3.4. "Taylor series" for $\mathcal{F}$-tensorial cochains. For a general 0 -cochain $H \in \mathcal{D}=$ $C^{0}(\mathcal{D}, \mathcal{D}), \delta H$ is a 1 -cochain with $\delta H(K)=[K, H]$. In the case of the $\mathcal{F}$-tensorial 0 -cochains consisting of the coordinate functions $x^{i}$ we have the 1-cochains $\delta x^{i}$ with $\left(\delta x^{i}\right)(K)=\left[K, x^{i}\right]$. We can also form composite cochains $\delta x^{i_{1}} \circ \delta x^{i_{2}} \circ \ldots \circ \delta x^{i_{|I|}}$ which are themselves 1-cochains. By 3.3 this composition is commutative and we may write

$$
\delta x^{i_{1}} \circ \ldots \circ \delta x^{i_{|I|}}=\delta x^{I} \text { with } I=\left\{i_{1}, \ldots, i_{|I|}\right\} .
$$


It can be shown that the action of $\delta x^{I}$ on $\partial_{J}$ is given by

$$
\delta x^{I}\left(\partial_{J}\right)= \begin{cases}0, & \text { if }|J|<|I| \\ \frac{1}{(|J|-|I|) !} \sum_{\sigma \in \mathcal{S}_{|J|}} \delta_{j_{\sigma_{1}}}^{i_{1}} \ldots \delta_{j_{|| I \mid}}^{i_{|I|}} \partial_{j_{\sigma_{|I|+1}} \ldots \partial_{j_{|J|}},} & \text { if }|J| \geq|I| .\end{cases}
$$

We may now write any $\mathcal{F}$-tensorial 1 -cochain as a series,

$$
\begin{aligned}
A & =A_{(\emptyset)} \delta x^{\emptyset}+A_{(i)} \delta x^{i}+\frac{A_{(i j)}}{2 !} \delta x^{i} \circ \delta x^{j}+\ldots+\frac{A_{(I)}}{|I| !} \delta x^{I}+\ldots \\
& =\sum_{I} \frac{1}{|I| !} A_{(I)} \delta x^{I}
\end{aligned}
$$

where $\delta x^{\emptyset}=1$, the identity cochain on $\mathcal{D}$. This is verified by checking that both sides have the same structure functions, as follows.

The action of the operator (12) on the unit function gives us the structure function of $\delta x^{I}$. We find that

$$
\left(\delta x^{I}\right)_{(J)}=0 \quad \text { if } \quad J \neq I
$$

and for $J=I$ (same unordered set of indices)

$$
\left(\delta x^{I}\right)_{(J)}=\sum_{\sigma \in \mathcal{S}_{|I|}} \delta_{j_{\sigma_{1}}}^{i_{1}} \ldots \delta_{j_{\sigma|I|}}^{i_{|I|}}
$$

Hence the structure function of

$$
\frac{1}{|I| !} A_{\left(i_{1} \ldots i_{|I|}\right)} \delta x^{I}
$$

is

$$
\begin{aligned}
\frac{1}{|I| !} A_{\left(i_{1} \ldots i_{|I|}\right)}\left(\delta x^{I}\right)_{(J)} & \left.=\frac{1}{|I| !} A_{\left(i_{1} \ldots i_{|I|}\right)} \sum_{\sigma \in \mathcal{S}_{|I|}} \delta_{\left(j_{\sigma_{1}}\right.}^{i_{1}} \ldots \delta_{j_{|| I \mid}}^{i_{|I|}}\right) \\
& =\frac{1}{|I| !} \sum_{\sigma \in \mathcal{S}_{|I|}} A_{\left(j_{1} \ldots j_{|I|}\right)}=A_{(J)}=A_{(I)}
\end{aligned}
$$

The series for any $\mathcal{F}$-tensorial $p$-cochain is

$$
A=\sum_{I_{1}, \ldots, I_{p}} \frac{1}{\prod_{i=1}^{p}\left|I_{i}\right| !} A_{\left(I_{1}, \ldots, I_{p}\right)} \delta x^{I_{1}} \cup \ldots \cup \delta x^{I_{p}} .
$$

Again each side has the same structure functions.

In (13) and (14) the coefficient functions $A_{(\ldots)}$ may be chosen arbitrarily. For example, in (13) one might take $A_{(I)}=0$ for $|I| \leq p-1$ and $A_{(I)} \neq 0$ for $|I|=p$. Then $A(H)=0$ for ord $H<p$; we say then that ord $A=p$ and find that the leading term in the Taylor series for $A$ is $A_{\left(i_{1}, \ldots i_{p}\right)} \delta x^{i_{1}} \circ \ldots \circ \delta x^{i_{p}}$, where $A_{\left(i_{1}, \ldots, i_{p}\right)}$ is a symmetric covariant tensor field. This is the dual result to the well known fact that the coefficients of the leading terms of a linear differential operator make up a symmetric contravariant tensor field.

\section{The isomorphism between $C_{\mathcal{F}}^{*}(\mathcal{D}, \mathcal{D})$ and $J_{\Delta} C_{A S}^{*}$}

4.1. Introduction. We introduce a complex $\left(C_{A S}^{*}, \delta_{A S}\right)$ called the Alexander-Spanier $(A S)$-complex, [Sp], [Mas]. The elements of $C_{A S}^{p}$ are smooth, complex-valued functions each defined on some open neighbourhood of the diagonal subset $\Delta_{p+1}$ of $Q^{p+1}$, the 
Cartesian product of $p+1$ copies of $Q . C_{A S}^{*}$ has a product, called concatenation, [Coq], and written as a dot

$$
C_{A S}^{p} \times C_{A S}^{q} \rightarrow C_{A S}^{p+q}, \quad(F, G) \mapsto F \cdot G
$$

and a differential, $\delta_{A S}$ :

$$
\delta_{A S}: C_{A S}^{p} \rightarrow C_{A S}^{p+1}
$$

Together these make $\left(C_{A S}^{*}, \delta_{A S},.\right)$ into an associative differential graded algebra (DGalgebra).

We define a map

$$
\Phi: C_{A S}^{*} \rightarrow C_{\mathcal{F}}^{*}(\mathcal{D}, \mathcal{D})
$$

which depends only on the partial derivatives of the $A S$-cochains on the diagonal subset $\Delta_{p+1}=\{(x, x, \ldots, x): x \in Q\}$ of $Q^{p+1}$ and not elsewhere. With this in mind we use the $A S$-algebra to construct an associated DG-algebra $\left(J_{\Delta} C_{A S}^{*}, \delta_{A S},.\right)$ whose elements of degree $p$ are the jets of $A S$ - $p$-cochains which are evaluated on $\Delta_{p+1}$.

The main result of this chapter is that the map $\Phi$ gives an isomorphism between the DG-algebras $\left(J_{\Delta} C_{A S}^{*}, \delta_{A S},.\right)$ and $\left(C_{\mathcal{F}}^{*}(\mathcal{D}, \mathcal{D}), \delta, \cup\right)$.

Section 4.2 describes the $A S$-complex. Particular elements, called [BR] separable cochains, are introduced. In section 4.3 we define the map $\Phi: C_{A S}^{p} \rightarrow C_{\mathcal{F}}^{p}(\mathcal{D}, \mathcal{D})$, and show that $\Phi$ is a morphism of DG-algebras. It is not however injective. In section 4.4 we show that, for $F \in C_{A S}^{p}$, the differential operator $\Phi^{F}\left(H_{1}, \ldots, H_{p}\right)$ depends on $F$ only through the jet of $F$ on the diagonal subset $\Delta_{p+1} \subset M^{p+1}$. Two functions $F_{1}, F_{2} \in C_{A S}^{p}$ having the same jet on $\Delta_{p+1}$ but differing elsewhere will satisfy $\Phi^{F_{1}}=\Phi^{F_{2}}$. In section 4.5 we prove that $\Phi: J_{\Delta} C_{A S}^{*} \rightarrow C_{\mathcal{F}}^{*}(\mathcal{D}, \mathcal{D})$ is an isomorphism of DG-algebras.

The isomorphism gives a cross-fertilisation of the properties of the two types of cochain, $\mathcal{F}$-tensorial and Alexander-Spanier. Thus $A S$-cochains acquire a Lie derivative with respect to vector fields and an interior product with respect to functions, whilst $\mathcal{F}$-tensorial cochains gain a commutative product. Some details are given in section 4.6.

In section 4.7 the isomorphism is refined slightly to one between a subalgebra of $J_{\Delta} C_{A S}^{*}$ consisting of jets of what we call normalised $A S$ cochains and the subalgebra $C^{*}(\mathcal{D}, \mathcal{F}, \mathcal{D}) \subset C_{\mathcal{F}}^{*}(\mathcal{D}, \mathcal{D})$ of normalised $\mathcal{F}$-tensorial cochains.

\subsection{The Alexander-Spanier complex}

Definition. An $A S$-p-cochain is an element of $C^{\infty}\left(U_{p+1}, \mathbf{C}\right)$ where $U_{p+1}$ is an open neighbourhood of the diagonal $\Delta_{p+1} \subset Q^{p+1}, \Delta_{p+1}=\{(x, x, \ldots, x): x \in Q\}$. The vector space

$$
C_{A S}^{*}=\sum_{p=0}^{\infty} C_{A S}^{p}
$$

is made into an algebra by defining the concatenation product:

For $F \in C_{A S}^{p}, G \in C_{A S}^{q}$ the product $F . G \in C_{A S}^{p+q}$ is defined by

$$
(F . G)\left(x_{0}, \ldots, x_{p}, x_{p+1}, \ldots, x_{p+q}\right)=F\left(x_{0}, \ldots, x_{p}\right) G\left(x_{p}, \ldots, x_{p+q}\right) .
$$


The Alexander-Spanier differential $\delta_{A S}: C_{A S}^{p} \rightarrow C_{A S}^{p+1}$ is defined by

$$
\left(\delta_{A S} F\right)\left(x_{0}, . ., x_{p+1}\right)=\sum_{j=0}^{p+1}(-1)^{j} F\left(x_{0}, \ldots, \hat{x}_{j}, \ldots, x_{p+1}\right)
$$

where $\hat{x}_{j}$ denotes omission of $x_{j}$.

There are particular sorts of $A S$-cochains called separable cochains which are of the form

$$
F=f_{0} \otimes f_{1} \otimes \ldots \otimes f_{p} \in \mathcal{F}^{\otimes p+1}
$$

That is,

$$
F\left(x_{0}, \ldots, x_{p}\right)=f_{0}\left(x_{0}\right) f_{1}\left(x_{1}\right) \ldots f_{p}\left(x_{p}\right) .
$$

For separable $F=f_{0} \otimes \ldots \otimes f_{p} \in C_{A S}^{p}$ and $G=g_{0} \otimes \ldots \otimes g_{q} \in C_{A S}^{q}$ we have

$$
F . G=f_{0} \otimes \ldots \otimes f_{p} g_{0} \otimes \ldots \otimes g_{q}
$$

and

$$
\begin{aligned}
\delta_{A S} F= & \left(1 \otimes f_{0} \otimes \ldots \otimes f_{p}\right)-\left(f_{0} \otimes 1 \otimes f_{1} \otimes \ldots \otimes f_{p}\right) \\
& +\ldots+(-1)^{p+1}\left(f_{0} \otimes \ldots \otimes f_{p} \otimes 1\right) .
\end{aligned}
$$

Every $F \in C_{A S}^{p}$ is the limit of a sequence of sums of separable functions. If a conjecture holds for separable functions in $C_{A S}^{p}$ then it follows from linearity and continuity that the conjecture will hold true for all elements of $C_{A S}^{p}$.

4.3. The map $\Phi: C_{A S}^{p} \rightarrow C_{\mathcal{F}}^{p}(\mathcal{D}, \mathcal{D})$. For $F \in C_{A S}^{p}, H_{j} \in \mathcal{D}, \psi \in \mathcal{F}$ we define $\Phi^{F} \in C_{\mathcal{F}}^{p}(\mathcal{D}, \mathcal{D})$ by $[\mathrm{HB}, \mathrm{BR}]$

$$
\begin{gathered}
\left(\Phi^{F}\left(H_{1}, \ldots, H_{p}\right) \psi\right)\left(x_{0}\right) \\
=\left[H_{1}\left(x_{1}\right)\left[H_{2}\left(x_{2}\right)\left[\ldots\left[H_{p}\left(x_{p}\right)\left[F\left(x_{0}, \ldots, x_{p}\right) \cdot \psi\left(x_{p}\right)\right]\right]_{x_{p}=x_{p-1}}\right] \ldots\right]_{x_{2}=x_{1}}\right]_{x_{1}=x_{0}} .
\end{gathered}
$$

The cochain $\Phi^{F}$ is evidently $\mathcal{F}$-tensorial.

For separable $F$ the equation (15) simplifies to

$$
\Phi^{F}\left(H_{1}, \ldots, H_{p}\right)=\Phi^{f_{0} \otimes \ldots \otimes f_{p}}\left(H_{1}, \ldots, H_{p}\right)=f_{0} H_{1} f_{1} \ldots H_{p} f_{p} \in \mathcal{D} .
$$

Again, for separable $F$,

$$
\begin{aligned}
& \Phi^{\delta_{A S} F}\left(H_{1}, \ldots, H_{p+1}\right) \\
& =\left(\left(1 \otimes f_{0} \otimes \ldots \otimes f_{p}\right)-\left(f_{0} \otimes 1 \otimes \ldots f_{p}\right)+\ldots+(-1)^{p+1}\left(f_{0} \otimes \ldots \otimes f_{p} \otimes 1\right)\right)\left(H_{1}, \ldots, H_{p}\right) \\
& =1 H_{1} f_{0} H_{2} \ldots H_{p+1} f_{p}-f_{0} H_{1} 1 H_{2} \ldots H_{p+1} f_{p}+\ldots+(-1)^{p+1} f_{0} H_{1} \ldots f_{p} H_{p+1} \\
& =H_{1} f_{0} H_{2} \ldots H_{p+1} f_{p}-f_{0} H_{1} H_{2} f_{1} \ldots H_{p+1} f_{p}+\ldots+(-1)^{p+1} f_{0} H_{1} \ldots f_{p} H_{p+1} \\
& =\delta \Phi^{F}\left(H_{1}, \ldots, H_{p+1}\right) .
\end{aligned}
$$

So $\Phi^{\delta_{A S} F}=\delta \Phi^{F}$ for separable functions $F$ and hence by linearity and continuity for all $F \in C_{A S}^{p}$. In a similar way, for $F \in C_{A S}^{p}$ and $G \in C_{A S}^{q}$, both separable, one may verify that

$$
\Phi^{F . G}\left(H_{1}, \ldots, H_{p+q}\right)=\left(\Phi^{F} \cup \Phi^{G}\right)\left(H_{1}, \ldots, H_{p+q}\right) .
$$

Hence $\Phi^{F . G}=\Phi^{F} \cup \Phi^{G}$ for separable functions and therefore for all $C_{A S}^{*}$. 
4.4. $\Phi$ factors through $J_{\Delta} C_{A S}^{*}$. In this section we prove that for $F \in C_{A S}^{p}$, $\Phi^{F}\left(H_{1}, \ldots H_{p}\right)$ depends on $F$ only through the jet of $F$ at the diagonal $\Delta_{p+1} \subset Q^{p+1}$. We denote by $J_{\Delta} C_{A S}^{*}$ the set of jets on $\Delta_{p+1}$ of elements $F \in C_{A S}^{p}$.

Notation. The point $x_{k} \in Q$ has coordinates $x_{k}^{i}$. Denote

$$
\frac{\partial}{\partial x_{k}^{i}} F\left(x_{0}, \ldots, x_{k}, \ldots, x_{p}\right) \text { by } F_{, k^{i}}
$$

and

$$
\frac{\partial}{\partial x_{k}^{i_{1}}} \frac{\partial}{\partial x_{k}^{i_{2}}} \ldots \frac{\partial}{\partial x_{k}^{i_{|I|}}} F\left(x_{0}, \ldots, x_{k}, \ldots, x_{p}\right)=\left(\frac{\partial}{\partial x_{k}}\right)^{I} F\left(x_{0}, \ldots, x_{k}, \ldots, x_{p}\right)
$$

by

$$
F_{, k^{I}}\left(x_{0}, \ldots, x_{k}, \ldots, x_{p}\right)
$$

where $I=\left\{i_{1}, \ldots, i_{|I|}\right\}$.

We shall often need to set several of the arguments $x_{k}$ of $F$ as equal and then to differentiate $F$ with respect to the coordinate $x_{k}^{i}$. For example if $F \in C_{A S}^{2}$, then by the chain rule

$$
\begin{gathered}
\frac{\partial}{\partial x_{1}^{i}} F\left(x_{0}, x_{1}, x_{1}\right)=F_{, 1^{i}}\left(x_{0}, x_{1}, x_{1}\right)+F_{, 2^{i}}\left(x_{0}, x_{1}, x_{1}\right) \equiv F_{,(1+2)^{i}}\left(x_{0}, x_{1}, x_{1}\right) \\
\left(\frac{\partial}{\partial x_{1}}\right)^{I} F\left(x_{0}, x_{1}, x_{1}\right)=F_{,(1+2)^{i_{1}}(1+2)^{i_{2}} \ldots(1+2)^{i}|I|}\left(x_{0}, x_{1}, x_{1}\right) \\
\equiv F_{,(1+2)^{I}}\left(x_{0}, x_{1}, x_{1}\right) .
\end{gathered}
$$

With this notation we may use (15) with $\psi=1$ to write the structure function of $\Phi^{F}$ as

$$
\begin{aligned}
\Phi_{\left(I_{1}, I_{2}\right)}^{F}\left(x_{0}\right) & =\left[\frac{\partial}{\partial x_{1}^{I_{1}}}\left(\left[\frac{\partial}{\partial x_{2}^{I_{2}}} F\left(x_{0}, x_{1}, x_{2}\right)\right]_{x_{2}=x_{1}}\right)\right]_{x_{1}=x_{0}} \\
& =F_{,(1+2)^{I_{1} 2^{I_{2}}}}\left(x_{0}, x_{0}, x_{0}\right) .
\end{aligned}
$$

Similarly one may show that for $F \in C_{A S}^{p}$,

$$
\Phi_{\left(I_{1}, \ldots I_{p}\right)}^{F}\left(x_{0}\right)=F_{,(1+\ldots+p)^{I_{1}}(2+\ldots+p)^{I_{2}} \ldots p^{I_{p}}}\left(x_{0}, \ldots x_{0}\right)
$$

which is a combination of jets of $F$ on the diagonal $\Delta$.

4.5. $\Phi:\left(J_{\Delta} C_{A S}^{*}, \delta_{A S},.\right) \rightarrow\left(C_{\mathcal{F}}^{*}(\mathcal{D}, \mathcal{D}), \delta, \cup\right)$ is an isomorphism

4.5.1. Injectivity of $\Phi: J_{\Delta} C_{A S}^{*} \rightarrow C_{\mathcal{F}}^{*}(\mathcal{D}, \mathcal{D})$. We have seen that the $\mathcal{F}$-tensorial cochain $\Phi^{F}$ depends only on the jet of $F$ at $\Delta$. The jets on the diagonal inherit from $C_{A S}^{*}$ the differential, grading and concatenation product structures, so they themselves form a DG-algebra, $\left(J_{\Delta} C_{A S}^{*}, \delta_{A S},.\right)$ We next show that if we restrict $\Phi$ to these (infinite-order) jets, then $\Phi$ becomes injective.

For this, we must show that if $\Phi^{F}=\Phi^{G}$ then $F$ and $G$ have the same jets on $\Delta$. Now if $\Phi^{F}=\Phi^{G}$ they must have the same structure functions, which implies [BR] that their jets on $\Delta$ are equal. 
4.5.2. Surjectivity of $\Phi: J_{\Delta} C_{A S}^{*} \rightarrow C_{\mathcal{F}}^{*}(\mathcal{D}, \mathcal{D})$. We have shown that $\Phi$ is an injective homomorphism. To show that $\Phi$ is an isomorphism we must produce

$$
\Phi^{-1}: C_{\mathcal{F}}^{*}(\mathcal{D}, \mathcal{D}) \rightarrow J_{\Delta} C_{A S}^{*}, \quad A \mapsto F^{A} .
$$

That is, given $A \in C_{\mathcal{F}}^{p}(\mathcal{D}, \mathcal{D})$ we must construct the jets on $\Delta^{p+1}$ of a function $F^{A} \in C_{A S}^{p}$ so that $\Phi^{F^{A}}=A$.

For $p=0$ we have $C_{\mathcal{F}}^{0}(\mathcal{D}, \mathcal{D})=\mathcal{F}$ and $F^{A}=A$.

For $p>0$ the cochain $A \in C_{\mathcal{F}}^{p}(\mathcal{D}, \mathcal{D})$ has structure function $A_{\left(I_{1}, \ldots, I_{p}\right)}(x)$. Now $F \in$ $C_{A S}^{p}$ has Taylor series

$$
F\left(x_{0}, \ldots x_{p}\right)=\sum_{I_{1}} \ldots \sum_{I_{p}}\left(\prod_{k=1}^{p}\left|I_{k}\right| !\right)^{-1} F_{, 1^{I_{1}} \ldots p^{I_{p}}}\left(x_{0}, \ldots, x_{0}\right)\left(x_{1}-x_{0}\right)^{I_{1}} \ldots\left(x_{p}-x_{0}\right)^{I_{p}} .
$$

One can show $[\mathrm{BR}]$ that by setting

$$
\begin{aligned}
F_{, 1} I_{1} \ldots p^{I_{p}} & \left(x_{0}, x_{0}, x_{0}\right) \\
& =(-1)^{\left|I_{12}\right|+\ldots+\left|I_{p 2}\right|} \sum_{I_{11} \cup I_{12}=I_{1}} \ldots \sum_{I_{p-1,1} \cup I_{p-1,2}=I_{p-1}} A_{\left(I_{11}, I_{12} \cup I_{21}, \ldots, I_{p-1,2} \cup I_{p}\right)}\left(x_{0}\right)
\end{aligned}
$$

we obtain $\Phi^{F^{A}}=A$. Thus $\Phi$ is an isomorphism. The convergence of the above formal series is not discussed but we invoke Borel's Lemma [Gib] that a $C^{\infty}$ function on $Q^{p+1}$ which has the above jets does exist.

4.6. Additional structures on $C_{\mathcal{F}}^{*}(\mathcal{D}, \mathcal{D})$ and $C_{A S}^{*}$. Since $C_{\mathcal{F}}^{p}(\mathcal{D}, \mathcal{D})$ and $J_{\Delta} C_{A S}^{p}$ are isomorphic DG-algebras, any structure admitted by $C_{\mathcal{F}}^{p}(\mathcal{D}, \mathcal{D})$ will give a corresponding structure to $C_{A S}^{p}$ (or at least to $J_{\Delta} C_{A S}^{p}$ ) and vice versa.

4.6.1. Composition of cochains in $C_{A S}^{*}$. For $\theta \in C_{\mathcal{F}}^{1}(\mathcal{D}, \mathcal{D}), A \in C_{\mathcal{F}}^{p}(\mathcal{D}, \mathcal{D})$ the composition $\theta \circ A \in C_{\mathcal{F}}^{p}(\mathcal{D}, \mathcal{D})$ induces the composition of $A S$-cochains

$$
(F \circ G)\left(x_{0}, \ldots, x_{p}\right)=F\left(x_{0}, x_{p}\right) G\left(x_{0}, \ldots, x_{p}\right)
$$

which might be called the "encircle" product of an $A S$ - $p$-cochain by an $A S$-1-cochain. In particular if $F, G \in C_{A S}^{1}$ then $(F \circ G)\left(x_{0}, x_{1}\right)=F\left(x_{0}, x_{1}\right) G\left(x_{0}, x_{1}\right)$.

4.6.2. Commutative product of two $\mathcal{F}$-tensorial p-cochains. AS-cochains of the same order are real functions on the same space so can be multiplied to produce another $A S$-cochain of the same order. So therefore can $\mathcal{F}$-tensorial cochains.

Let $A, B \in C_{\mathcal{F}}^{p}(\mathcal{D}, \mathcal{D})$ have corresponding $A S$-cochains $F^{A}, F^{B}$,

$$
A=\Phi^{F^{A}}, \quad B=\Phi^{F^{B}} .
$$

Define the (commutative) product

$$
A B=B A=\Phi^{F^{A} F^{B}} \in C_{\mathcal{F}}^{p}(\mathcal{D}, \mathcal{D}) .
$$

For 1-cochains $\theta, \theta^{\prime}$ we have $\theta \theta^{\prime}=\theta \circ \theta^{\prime}$.

4.6.3. Interior product $\iota_{f}$ and Lie derivative $L_{V}$ of $A S$-cochains. We found in sections 2.5 and 2.6 that the interior product map $\iota_{K}: C^{p+1}(\mathcal{D}, \mathcal{D}) \rightarrow C^{p}(\mathcal{D}, \mathcal{D})$ preserves $\mathcal{F}$ tensoriality if and only if $K \in \mathcal{F}$, and the Lie derivative $L_{K}$ does so only if ord $K \leq 1$. 
The $A S$-cochains inherit these structures as follows:

$$
\begin{aligned}
\left(\iota_{f} F\right)\left(x_{0}, \ldots, x_{p}\right) & =\sum_{j=0}^{p}(-1)^{j} f\left(x_{j}\right) F\left(x_{0}, \ldots x_{j-1}, x_{j}, x_{j}, x_{j+1}, \ldots, x_{p}\right), \\
\left(L_{V} F\right)\left(x_{0}, \ldots, x_{p}\right) & =\sum_{j=0}^{p} V^{i}\left(x_{j}\right) \partial_{x_{j}^{i}} F\left(x_{0}, \ldots, x_{p}\right) .
\end{aligned}
$$

4.7. Normalisation. An $A S$-cochain $F$ is said to be normalised if it vanishes when two contiguous variables are identified, i.e.

$$
F\left(x_{0}, \ldots, x_{j}, x_{j}, x_{j+2} \ldots, x_{p}\right)=0 .
$$

It can be shown that the jets of the normalised $A S$-cochains correspond isomorphically to the normalised $\mathcal{F}$-tensorial cochains.

5. From de Rham forms to Alexander-Spanier cochains. For a given $\omega \in$ $\Omega^{p}(M),[\mathrm{HB}]$ construct a function $F^{\omega}: \mathcal{V}_{p+1} \rightarrow \mathbf{R}$ where $\mathcal{V}_{p+1} \subset Q^{p+1}$ is a certain neighbourhood of the diagonal subset $\Delta_{p+1}=\{(x, \ldots, x): x \in Q\} \subset Q^{p+1}$ which is specified below. So, $\mathcal{V}_{p+1}$ consists of $(p+1)$-tuples $\left(x_{0}, \ldots, x_{p}\right) \in Q^{p+1}$ which are "close" to $\Delta_{p+1}$. This means that the $x_{j}$ in $Q$ are all reasonably close to each other.

The construction uses the geodesic paths of a connection $\Gamma$ on $T Q$, so we suppose that we are given this path structure.

At each point $x_{0} \in Q$ we take a convex normal neighbourhood $\mathcal{V}_{x_{0}}$ of $x_{0}$ ([CP], chap. 11). Then $\mathcal{V}_{x_{0}}$ is such that there is one and only one segment of a geodesic path joining any two points in $\mathcal{V}_{x_{0}}$. We define

$$
\mathcal{V}_{p+1}=\left\{\left(x_{0}, \ldots, x_{p}\right) \in Q^{p+1}: x_{1}, \ldots, x_{p} \in \mathcal{V}_{x_{0}}\right\}
$$

For $\left(x_{0}, \ldots x_{p}\right) \in \mathcal{V}_{p+1}$, we construct a $p$-simplex $S_{p}^{\Gamma}\left(x_{0}, \ldots, x_{p}\right)$ having vertices $x_{0}, \ldots, x_{p}$ as follows.

The edge $S_{1}^{\Gamma}\left(x_{i}, x_{j}\right), i<j$, of the $p$-simplex $S_{p}^{\Gamma}\left(x_{0}, \ldots, x_{p}\right)$ is the oriented geodesic path from $x_{i}$ to $x_{j}$. The 2-face $S_{2}^{\Gamma}\left(x_{i}, x_{j}, x_{k}\right), i<j<k$, is built by joining $x_{i}$ to points on $S_{1}^{\Gamma}\left(x_{j}, x_{k}\right)$ by geodesic paths.

3 -faces $S_{3}^{\Gamma}\left(x_{i}, x_{j}, x_{k}, x_{l}\right), i<j<k<l$, are formed by joining $x_{i}$ by geodesic paths to the points of $S_{2}^{\Gamma}\left(x_{j}, x_{k}, x_{l}\right)$ and so on.

It follows that

$$
\partial S_{p}\left(x_{0}, \ldots, x_{p}\right)=\sum_{j=0}^{p}(-1)^{j} S_{p-1}\left(x_{0}, \ldots, \hat{x}_{j}, \ldots, x_{p}\right) \equiv S_{p-1}\left(\partial\left(x_{0}, \ldots, x_{p}\right)\right) .
$$

Thus the map $F_{\Gamma}: \Omega^{*} \rightarrow N C_{A S}^{*}$ defined by

$$
F_{\Gamma}^{\omega}\left(x_{0}, \ldots, x_{p}\right)=\int_{S_{p}^{\Gamma}\left(x_{0}, \ldots, x_{p}\right)} \omega
$$

satisfies

$$
F_{\Gamma}^{d \omega}=\delta_{A S} F_{\Gamma}^{\omega}
$$


One can then show $[\mathrm{HB}]$ that, for $\Phi$ as defined in Chapter 4 ,

$$
\Phi_{\Gamma}^{F^{\omega}}\left(X_{1}, \ldots, X_{p}\right)=\frac{1}{p !} \omega\left(X_{1}, \ldots, X_{p}\right)
$$

i.e. the action of $\Phi_{\Gamma}^{F^{\omega}}$ on vector fields is independent of $\Gamma$ and agrees up to scale with $\omega$. In particular for $\theta \in \Omega^{1}$,

$$
\Phi_{\Gamma}^{F^{\theta}} \mid \mathcal{X}=\theta
$$

In order to evaluate the 1-cochain $\Phi_{\Gamma}^{F^{\theta}}$ on differential operators of order higher than 1 we need to know the structure functions of the cochain, or equivalently, the jets of the function $F_{\Gamma}^{\theta}\left(x_{0}, x_{1}\right)$ at $x_{1}=x_{0}$. The first few are

$$
\begin{aligned}
F_{\Gamma, 1^{a}}^{\theta}\left(x_{0}, x_{0}\right) & =\theta_{a}\left(x_{0}\right), \\
F_{\Gamma, 1^{a} 1^{b}}^{\theta}\left(x_{0}, x_{0}\right) & =\theta_{(a, b)}\left(x_{0}\right), \\
F_{\Gamma, 1^{a} 1^{b} 1^{c}}^{\theta} & =\theta_{(a, b c)}+\frac{1}{2} d \theta_{i,(a} \Gamma_{b c)}^{i}, \\
F_{\Gamma, 1^{a} 1^{b} 1^{c} 1^{d}}^{\theta} & \left.=\theta_{(a, b c d)}+\left[d \theta_{i,(a} \Gamma_{b c}^{i}\right], d\right) .
\end{aligned}
$$

where curved brackets ( ) around subscripts indicate that they are symmetrised. A general formula exists for the covariant derivatives of $F_{\Gamma}^{\theta}$. It is $[\mathrm{Ha}]$

$$
F_{\Gamma ;\left(1^{\left.a_{1} \ldots 1^{a_{p}}\right)}\right.}^{\theta}\left(x_{0}, x_{0}\right)=\theta_{\left(a_{1} ; a_{2} \ldots a_{p}\right)}\left(x_{0}\right) .
$$

It is evident from its construction that the $p$-simplex $S_{p}^{\Gamma}\left(x_{0}, \ldots, x_{p}\right)$ collapses to the $(p-1)$-simplex $S_{p-1}^{\Gamma}\left(x_{0}, \ldots \hat{x}_{j}, x_{j+1}, \ldots, x_{p}\right)$ whenever $x_{j+1}$ is set equal to $x_{j}$. The functions $F_{\Gamma}^{\omega}\left(x_{0}, \ldots, x_{p}\right)$ defined on $\mathcal{V}_{p+1}$ are thus normalised $A S$-cochains, and the cochains $\Phi^{F_{\Gamma}^{\omega}}$ are $\mathcal{F}$-relative.

6. Applications. Having forged our small Nothung let us bully some small dragons with it.

6.1. The probability current in quantum mechanics. We shall use the framework of the standard quantum mechanics of a system having configuration space $Q$ with half density wave functions $\psi$ such that $\int_{Q}|\psi|^{2}<\infty$. We suppose that we are given a Hamiltonian. This is a symmetric operator $H \in \mathcal{D}$ which possesses a self adjoint extension. The time development of $\psi$ is then specified by the Schrödinger equation

$$
i \partial_{t} \psi=H \psi
$$

The amplitude density $\rho_{(\phi, \psi)}=\bar{\phi} \psi$ for two wave functions $\phi, \psi$ satisfies

$$
\int_{Q} \partial_{t} \rho_{(\phi, \psi)}=0
$$

which implies that we may write

$$
\partial_{t} \rho_{(\phi, \psi)}=-\operatorname{div} J_{(\phi, \psi)}
$$

for some transition probability flux vector field, or current. This fixes $\operatorname{div} J$, not $J$ itself, though some textbooks claim that for $Q=\mathbf{R}^{\mathbf{3}}$ and $H=-\frac{1}{2} \nabla^{2}+V(\mathbf{r})$, the "correct" $J$ among those with the right divergence is

$$
J_{(\phi, \psi)}=\Im(\bar{\phi} \nabla \psi) .
$$


We investigate what structure is required to select the "correct" $J$ when $H$ is an arbitrary Hamiltonian operator on an arbitrary configuration manifold $Q$.

We first recall the properties of de Rham $p$-currents-linear complex-valued functions on "test" $p$-forms on $Q,[\mathrm{deR}]$. A 0 -current $\rho$ is a scalar density,

$$
\rho(f)=\int_{Q} f \rho, \quad f \in \mathcal{F} .
$$

A 1-current $J$ is a vector density. It acts on test 1-forms

$$
J(\theta)=\int_{Q} \theta_{i} J^{i}, \quad \theta \in \Omega^{1} .
$$

There is a natural map div from $p$-currents to $(p-1)$-currents,

$$
(\operatorname{div} J)(\omega)=-J(d \omega), \quad \omega \in \Omega^{p-1} .
$$

We shall need to extend the notion of $p$-currents to be linear complex-valued functions on test $\mathcal{F}$-relative $p$-cochains; let $\mathcal{J}_{p}$ be the space of these $p$-currents. Copy the definition of div:

$$
\operatorname{div}: \mathcal{J}_{p} \rightarrow \mathcal{J}_{p-1}, \quad(\operatorname{div} J)(A)=-J(\delta A)
$$

where $\delta$ is the Hochschild differential. Then for $H \in \mathcal{D}, f \in \mathcal{F}=C^{0}(\mathcal{D}, \mathcal{F} ; \mathcal{D})$ and wave functions $\phi, \psi$ we have a 1-current

$$
\begin{gathered}
H_{(\phi, \psi)}(A)=\int_{Q} \bar{\phi} A(H) \psi, \quad A \in C^{1}(\mathcal{D}, \mathcal{F} ; \mathcal{D}), \\
\left(\operatorname{div} H_{(\phi, \psi)}\right)(f)=-H_{(\phi, \psi)}(\delta f)=-\int \bar{\phi} \delta f(H) \psi=-\int \bar{\phi}[H, f] \psi .
\end{gathered}
$$

Then treating $\rho_{(\phi, \psi)}$ as an element of $\mathcal{J}_{0}$, we obtain

$$
\left(\partial_{t} \rho_{(\phi, \psi)}\right)(f)=\partial_{t} \int_{Q} \bar{\phi} f \psi=i \int_{Q} \bar{\phi}[H, f] \psi=-\left(\operatorname{div} i H_{(\phi, \psi)}\right)(f) .
$$

So

$$
\partial_{t} \rho_{(\phi, \psi)}=-\operatorname{div} i H_{(\phi, \psi)}
$$

as an equation of two Hochschild 0 -currents. This suggests that $i H_{(\phi, \psi)}$ be regarded as the probability 1-current density. It is a Hochschild 1-current, not yet a de Rham 1-current.

To obtain a de Rham 1-current, which maps 1-forms into $\mathbf{C}$, we need a way to extend the domain of 1 -forms from vector fields to all of $\mathcal{D}$. But this map is to hand:

$$
D_{\Gamma}=\Phi \circ F_{\Gamma}: \Omega^{*} \rightarrow C^{*}(\mathcal{D}, \mathcal{F} ; \mathcal{D}), \quad \omega \mapsto \Phi^{F_{\Gamma}^{\omega}}=D_{\Gamma}^{\omega}
$$

satisfies $D_{\Gamma}^{d \omega}=\delta D_{\Gamma}^{\omega}$ and $\left.D_{\Gamma}^{\omega}\right|_{\mathcal{X}}=(p !)^{-1} \omega$. So we take the de Rham current to be

$$
J_{(\phi, \psi)}(\theta)=i H_{(\phi, \psi)}\left(D_{\Gamma}^{\theta}\right)=i \int_{Q} \bar{\phi} D_{\Gamma}^{\theta}(H) \psi
$$

One can check that for $Q=\mathbf{R}^{3}, H=-\frac{1}{2} \nabla^{2}+V(\mathbf{r}), \Gamma=$ Euclidean connection, and Cartesian coordinates $x^{i}$, we obtain the usual formula

$$
J_{(\phi, \psi)}(\theta)=-\frac{i}{2} \int_{Q} \theta_{i}\left(\bar{\phi} \partial_{i} \psi-\left(\partial_{i} \bar{\phi}\right) \psi\right) .
$$


6.2. An $\mathcal{F}$-relative metric 2-cochain for a Riemannian manifold. We now suppose that $Q$ possesses a Riemannian metric $g$. There are many $\mathcal{F}$-relative 2 -cochains on $\mathcal{D}$ whose restrictions to $\mathcal{X}$ coincide with the symmetric covariant tensor field $g$. If there were a natural choice for such a cochain then we could define an "inner product" $g\left(H_{1}, H_{2}\right) \in \mathcal{D}$ of order ord $H_{1}+$ ord $H_{2}-2$, perhaps extend the Levi-Civita covariant derivative from $\nabla_{X_{1}} X_{2}$ to $\nabla_{X_{1}} H_{2}$, and explore torsion and curvature in this wider context. In section 5.1 we showed how to obtain an $\mathcal{F}$-relative $p$-cochain from an antisymmetric covariant tensor field (a differential form) by integrating the form over a certain $p$-simplex. This trick is not available for symmetric covariant tensor fields, and we must try something else.

Instead of working with the partial differential operators $\partial_{I}$ of $(10)$ it is convenient to use the symmetrised covariant differential operators

$$
\nabla_{I}=\nabla_{\left(i_{1}, \ldots i_{p}\right)}, \nabla_{I} \psi=\psi_{;\left(i_{1} \ldots i_{p}\right)} .
$$

So

$$
\nabla_{i}=\partial_{i}, \nabla_{\left(i_{1}, i_{2}\right)}=\partial_{i_{1}} \partial_{i_{2}}-\Gamma_{i_{1} i_{2}}^{k} \partial_{k},
$$

and so forth. Note that $\nabla_{i j k} \neq \nabla_{i j} \nabla_{k}$.

Consider an $\mathcal{F}$-relative 1 -cochain $\theta$ of order 2 ; this kills differential operators of order 1 but spares operators of order 2 or more. The Taylor series (13) starts

$$
\theta=\theta_{(i j)} \partial x^{i} \circ \partial x^{j}+\theta_{(i j k)} \partial x^{i} \circ \partial x^{j} \circ \partial x^{k}+\ldots
$$

where the $\theta_{(i j)}=\theta\left(\partial_{i} \partial_{j}\right)$ are the components of a symmetric covariant tensor field. Let us choose one such cochain $\theta^{g}$ such that $\theta_{(i j)}^{g}=g_{i j}$. Postponing the choice of the higher coefficients in the Taylor series, we note that for $X, Y \in \mathcal{X}$,

$$
\begin{aligned}
\delta \theta^{g}(X, Y) & =X \circ \theta^{g}(Y)-\theta^{g}(X Y)+\theta^{g}(X) \circ Y \\
& =-\theta^{g}\left(X^{i} \partial_{i} \circ Y^{j} \partial_{j}\right)=-X^{i} Y^{j} \theta^{g}\left(\partial_{i} \partial_{j}\right)=-g(X, Y) .
\end{aligned}
$$

For ord $H=2, H=h^{i j} \partial_{i} \partial_{j}+h^{i} \partial_{i}+h^{\emptyset}$, we have

$$
\theta^{g}(H)=g_{i j} \partial x^{i} \circ \partial x^{j}(H)=h^{i j} g_{i j}=h_{i}^{i} .
$$

So $\theta^{g}$ is a sort of trace; not a true trace because usually $\theta^{g}\left(\left[H_{1}, H_{2}\right]\right) \neq 0$. We now fix the higher coefficients of the Taylor series. Instead of the structure functions $\theta_{(I)}^{g}=$ $\theta^{g}\left(\partial_{I}\right) 1$ used earlier, we replace $\partial_{I}$ by $\nabla_{(I)}$ and consider the "symmetrised covariant structure functions" $\theta^{g}\left(\nabla_{\left(i_{1} \ldots i_{p}\right)}\right) 1$. For fixed $p$ these functions make up the components of a symmetrical covariant tensor field, since the functions $\theta^{g}\left(h^{i_{1} \ldots i_{p}} \nabla_{\left(i_{1} \ldots i_{p}\right)}\right) 1=$ $h^{i_{1} \ldots i_{p}} \theta^{g}\left(\nabla_{\left(i_{1} \ldots i_{p}\right)}\right) 1$ are scalar. We may therefore impose the condition that the tensor components $\theta^{g}\left(\nabla_{(I)}\right) 1$ vanish for all $|I|>2$. This determines the higher Taylor coefficients of $\theta^{g}$; for example $\theta_{(i j k)}^{g}=\theta^{g}\left(\partial_{i} \partial_{j} \partial_{k}\right) 1=3 \Gamma_{(i . j k)}$, the symmetrised Christoffel symbols. It follows that

$$
\theta^{g}\left(h^{i_{1} \ldots i_{p}} \nabla_{\left(i_{1} \ldots i_{p}\right)}\right)=\left(\begin{array}{c}
p \\
2
\end{array}\right) h_{a}^{a i_{1} \ldots i_{p-2}} \nabla_{\left(i_{1} \ldots i_{p-2}\right)} .
$$

We may now define the metric 2-cochain $g$ to be $-\delta \theta^{g}$ on all $\mathcal{D}$. It is natural to enquire whether we may define the covariant derivative of a differential operator. For 
$W \in \mathcal{X}, H \in \mathcal{D}$, can we define $\nabla_{W} H$ so that

$$
\begin{aligned}
\nabla_{W} & : \mathcal{D}_{p} \rightarrow \mathcal{D}_{p}, \\
\nabla_{f W} & =f \nabla_{W}, \\
\nabla_{W}(f H) & =W(f) \circ H+f \nabla_{W} H, \\
\nabla_{W}\left(g\left(H_{1}, H_{2}\right)\right) & =g\left(\nabla_{W} H_{1}, H_{2}\right)+g\left(H_{1}, \nabla_{W} H_{2}\right) ?
\end{aligned}
$$

The fourth condition is clearly impossible since the first two terms are $\mathcal{F}$-linear in $W$, but the third is not, when ord $H_{1} \geq 2$. So let us replace $\left(P 4^{\prime}\right)$ by the analogous condition for the Hochschild potential $\theta^{g}$ of the metric $g$,

$$
\nabla_{W}\left(\theta^{g}(H)\right)=\theta^{g}\left(\nabla_{W} H\right) .
$$

One may deduce from $(P 4)$ that

$$
\nabla_{i}\left(\nabla_{\left(j_{1} \ldots j_{p}\right)}\right)=p \mathcal{S}_{j_{1} \ldots j_{p}} \Gamma_{i j_{1}}^{a} \nabla_{\left(j_{2} \ldots j_{p} a\right)} .
$$

Here $\mathcal{S}_{j_{1} \ldots j_{p}}$ denotes symmetrisation over the indices $j_{1}, \ldots, j_{p}$. This in turn implies that

$$
\nabla_{W}\left(h^{j_{1} \ldots j_{p}} \nabla_{\left(j_{1} \ldots j_{p}\right)}\right)=W^{i} h_{; i}^{j_{1} \ldots j_{p}} \nabla_{\left(j_{1} \ldots j_{p}\right)}
$$

as one might naively expect. So we adopt (19) as our definition of $\nabla_{W}$ on $\mathcal{D}$.

\section{References}

[BR] F. J. Bloore and G. Roberts, The structure of $\mathcal{F}$-tensorial cochains of differential operators, Differential Geom. Appl. 10 (1999), 295-301.

[Coq] R. Coquereaux, Non-commutative geometry and theoretical physics, J. Geom. Phys. 6 (1989), 425-490.

[CP] M. Crampin and F. A. E. Pirani, Applicable Differential Geometry, Cambridge Univ. Press, 1986.

[deR] G. de Rham, Variétés différentiables, Hermann, 1955.

[Gib] C. G. Gibson, Singular Points of Smooth Mappings, Pitman, London, 1979.

[Ha] T. J. Harding, Ph.D. thesis, Liverpool University, 1990.

[HB] T. J. Harding and F. J. Bloore, Isomorphism of de Rham cohomology and relative Hochschild cohomology of differential operators, Annales Inst. H. Poincaré 58 (1993), 433-452.

[Ho1] G. Hochschild, On the cohomology groups of an associative algebra, Ann. Math. 46 (1945), 58-67.

[Ho2] G. Hochschild, Relative homological algebra, Trans. Amer. Math. Soc. 82 (1956), 246269.

[Mas] W. S. Massey, Homology and Cohomology Theory, Dekker, 1978.

[Sp] E. H. Spanier, Algebraic Topology, McGraw-Hill, 1966. 